\title{
Resposta assistencial de um serviço docente assistencial de APS à pandemia do COVID-19
}

\author{
Claunara Schilling Mendonça, Idiane Rosset, Marcelo Rodrigues Gonçalves, Cynthia Goulart \\ Molina Bastos, Adriana Farias de Medeiros, Alice Venturini Dias, Filipe Rodrigues \\ Nascimento, Franciele de Souza Masiero, Geferson Pelegrini, Jean Maito Ragnini, Lucas \\ Oliveira Leite, Nara Monte Arruda, Patricia de Freitas, Rafael Ballester Firmino, Ricardo \\ Pretto Reolom, Rodrigo Pedroso Tolio, George Henrique Aliatti Mantese
}

\section{RESUMO}

As respostas dos países à pandemia demonstram diferenças entre seus sistemas de saúde, tanto do ponto de vista do seu financiamento, da cobertura dos serviços, do modelo de atenção, como em relação às diferentes relações do nível central com as instâncias infraregionais. Esse relato descreve a resposta de um serviço de APS em Porto Alegre/RS, ligado a um Hospital de referência para o COVID-19, que é responsável por uma população de $\mathbf{4 3}$ mil pessoas (IBGE) a partir de 4 equipes de saúde da família.

Palavras-chave: COVID-19 , Pandemia, Atenção Primária à Saúde.

\section{ABSTRACT}

The responses of countries to the pandemic demonstrate differences between their health systems, both in terms of their financing, the coverage of services, the model of care, and in relation to the relationship between the central level with the infra-regional bodies. This report describes the response of a PHC service in Porto Alegre- RS, connected to a reference hospital for COVID-19, which is responsible for a population of 43 thousand people (IBGE).

Keywords: COVID-19, Pandemic, Primary Health Care.
Revista da Rede APS 2020

Publicada em: 15/04/2020

DOI:10.14295/aps.v2i1.63

Claunara Schilling Mendonça Idiane Rosset

Marcelo Rodrigues Gonçalves

(Universidade Federal do Rio Grande do Sul, HCPA, Rio Grande do Sul, Brasil)

Cynthia Goulart Molina

(UBS Santa Cecilia, Hospital de Clínicas de Porto Alegre- HCPA, Rio Grande do Sul, Brasil)

Adriana Farias de Medeiros

Alice Venturini Dias

Filipe Rodrigues Nascimento

Franciele de Souza Masiero

Geferson Pelegrini

Jean Maito Ragnini

Lucas Oliveira Leite

Nara Monte Arruda

Patricia de Freitas

Rafael Ballester Firmino

Ricardo Pretto Reolom

(Residentes HCPA, Brasil);

Rodrigo Pedroso Tolio

(SMS, Porto Alegre, Rio Grande do Sul, Brasil);

George Henrique Aliatti Mantese

(Telessaúde RS, Brasil)

Correspondência para:

Claunara Schilling Mendonça

claunara@ghc.com.br 
Mendonça, C. S.; Rosset, I.; Gonçalves, M. R.; Bastos, C. G. M.; Medeiros, A. F.; Dias, A. V.; Nascimento, F. R.; Pereira, F. V. P.; Pelegrini, G.; Pagnini J.; Leite, L. O.; Arruda, N. M.; Freitas, P.; Firmino, R. B.; Reolom, R.; Tolio, R. P.; Mantese, G. H. A.

\section{INTRODUÇÃO}

As respostas dos países à pandemia demonstram diferenças entre seus sistemas de saúde, tanto do ponto de vista do seu financiamento, da cobertura dos serviços, do modelo de atenção, como em relação às diferentes relações do nível central com as instâncias infraregionais. (Lorenzo, 2020; Liu, 2020; Kidd, 2020)

No Brasil, com seu Sistema Único de Saúde e com a Estratégia de Saúde da Família, a prática da Atenção Primária à Saúde é absolutamente central para a resposta local ao COVID-19 (Brasila, 2020). Esse relato descreve a resposta de um serviço de APS, ligado a um Hospital de referência para o COVID-19, na cidade de Porto Alegre/RS.

A Unidade Básica de Saúde Santa Cecília, é uma unidade docente assistencial, vinculada ao Hospital de Clínicas de Porto Alegre e à UFRGS, com os cursos de graduação de medicina, enfermagem, nutrição, fonoaudiologia, fisioterapia e psicologia. Localizado na Gerência Distrital Centro, é responsável por uma população de 43 mil pessoas (IBGE), com $18 \mathrm{mil}$ pessoas cadastradas e ativas, atendidas nos últimos 3 anos, tendo 20,7\% da população acima de 60 anos. É formada por 4 Equipes de Saúde da Família, conta com 10 ACS, duas nutricionistas, uma farmacêutica e uma assistente social. Conta com 12 professores de Medicina de Família e Comunidade, responsáveis pela graduação e pelo Programa de Residência Médica (06 R1, 02 R2 e 02 R3), 1 professora da nutrição e 2 professoras da enfermagem.

No dia dez de março, a partir do primeiro caso confirmado de COVID-19 em Porto Alegre, foi elaborado um Plano de Contingência para o enfrentamento da COVID-19, baseado em 4 princípios:

\section{I) PoRTA DE ENTRADA ESPECÍfICA PARA SINTOMÁTICO RESPIRATÓRIO (SR):}

Criado o fluxo especifico para sintomáticos respiratórios (SR) suspeitos de CO'VID-19, não tendo sido possível um circuito fechado de pacientes suspeitos - havia uma proposta inicial de construção de salas/tendas, na parte externa da unidade, específica para o COVID, que não pode ser realizada porque todo o serviço de engenharia estava envolvido na arnpliação de leitos de UTI, na área nova do HCPA. O fluxo dos sintomáticos respiratórios foi separado dos não sintomáticos, sem troca de funcionários ou pacientes entre as duas áreas. As equipes separadas visam diminuir o risco das instalações e dos profissionais disseminarem a contaminação e a equipe treinada desenvolve mais rapidamente o conhecimento individual e coletivo para lidar com pacientes corn COVID-19, e também diminui o volume de equipamento de proteção individual (EPI) necessário.

Reunião de alinhamento de fluxos com toda equipe médica, de enfermagem e pessoal da higienização para reforço do $\mathrm{EPI}$, repasse dos protocolos de atendimento, notificação de suspeitos, registros no prontuário, com complementação de dados clínicos registrados em fichas de papel. Os SR são avaliados no lado de fora da unidade, por residentes médicos, residente de enfermagem, residente da nutrição e alunos de medicina do último ano, priorizados por gravidade, suspeição de COVID19 recebimento de máscara e uma ficha numérica, sendo chamados para as três salas específicas para o atendimento de SR, atenididos pelos profissionais treinados e com EPI conforme recomendações. Nas salas de atendimento de suspeição do COVID19, realizadas por residentes de Medicina de Família e Comunidade (Brasilb, 2020), com proteção de contato e respiratória, foram realizadas coletas de swab e o seguimento de notificação, prescrição clínica e entrega presencial de medicamentos pelos médicos, sem circulação até a farmácia, com orientações por escrito para o isolamento 
ISSN 2596-3317 - DOI 10.14295/aps.v2i1.63

Mendonça, C. S.; Rosset, I.; Gonçalves, M. R.; Bastos, C. G. M.; Medeiros, A. F.; Dias, A. V.;

Nascimento, F. R.; Pereira, F. V. P.; Pelegrini, G.; Pagnini J.; Leite, L. O.; Arruda, N. M.; Freitas,

P.; Firmino, R. B.; Reolom, R.; Tolio, R. P.; Mantese, G. H. A.

domiciliar e atestado médico (também para contactantes, quando o caso).

Juntamente com as ações de acompanhamento por telefone, foi mantido um seguimento de todos os pacientes infectados, dos casos leves aos mais graves, identificando precocemente sinais de agravamento e encaminhamento ao serviço de emergência de referência, via SAMU (SMS/POA, 2020), e seguimento pós-alta desses pacientes e seus contactantes.

\section{II)PROTEÇÃO dOS}

\section{ProfissionaIS de SAÚde}

A fim de proteger a saúde física e mental dos profissionais envolvidos na atenção direta aos sintomáticos respiratórios, os fluxos foram sendo ajustados ao longo das semanas. Os registros dos atendimentos no sistema próprio da UBS/HCPA (AGHUSE) passaram a receber um registro específico, que é utilizado para notificação dos casos e telemonitoramento posterior. A documentação utilizada é previamente definida, sendo impressos todos os documentos utilizados na consulta do SR: atestados, prescrições, orientações para isolamento domiciliar. A circulação de pacientes foi definida da seguinte forma: SEM SINTOMAS RESPIRATÓRIOS: atendimento pelos doutorandos (alunos do quinto e sexto ano), via agenda de Demanda Espontânea / Acolhimento, com supervisão do professor/contratado responsável. COM SINTOMAS RESPIRATÓRIOS: suspeito de Covid 19 e demais pacientes com sintomas respiratórios e condições de espera (priorizar idosos e condições crônicas). Os pacientes são buscados na área externa pelo profissional que vai realizar o atendimento nas salas pré-determinadas. As equipes de trabalho foram organizadas em dois grupos para que não interagissem presencialmente.

Os profissionais da atenção tinham as normas de biossegurança reforçadas na supervisão dos casos, sendo responsáveis pelo cumprimento das regras de utilização dos EPIs, bem como pela limpeza de superfícies das salas (com luvas e desinfetantes) onde são realizados 0 atendimento, reforçada pela equipe de higienização no final de cada turno.

A fim de apoiar os profissionais de saúde a proteger suas famílias, foi negociado com o hospital a utilização de roupas do bloco cirúrgico (já que havia redução nas cirurgias eletivas) para todos os profissionais envolvidos no contato direto com os usuários. Além disso, foi disponibilizado um número de celular para atender todos os alunos de graduação, envolvidos na pandemia e, quando indicado, avaliados presencialmente e encaminhados para coleta do exame. Todos os profissionais envolvidos na atenção direta aos sintomáticos respiratórios tem livre acesso a aconselhamento e apoio psicológico, realizado por médica de família e comunidade contratada, responsável pela organização das atividades relacionadas ao atendimento dos sintomáticos respiratórios e do atendimento dos casos suspeitos do COVID 19.

\section{III)GARANTIA DOS CUIDADOS AOS PACIENTES NÃO-COVID-}

\section{DURANTE A PANDEMIA.}

Num primeiro momento, o serviço se concentrou na prevenção, contenção e atenção dos sintomáticos respiratórios, para, em seguida, seguir prestando o cuidado aos demais problemas de saúde que seguem afetando a população. Desde a primeira semana de atendimento dos casos da pandemia, as agendas eletivas foram bloqueadas e os pacientes acima de 60 anos pré-agendados foram contatados, com teleconsulta realizada pelos médicos residentes e doutorandos, supervisionados pelos supervisores, suas condutas registradas em planilha compartilhada. Na segunda semana, foi criada uma agenda específica para o teleatendimento, com a ampliação de números de telefone que foram disponibilizados para cada uma das 4 equipes de Saúde da Família. Esse agendamento 
ISSN 2596-3317 - DOI 10.14295/aps.v2i1.63

Mendonça, C. S.; Rosset, I.; Gonçalves, M. R.; Bastos, C. G. M.; Medeiros, A. F.; Dias, A. V.; Nascimento, F. R.; Pereira, F. V. P.; Pelegrini, G.; Pagnini J.; Leite, L. O.; Arruda, N. M.; Freitas, P.; Firmino, R. B.; Reolom, R.; Tolio, R. P.; Mantese, G. H. A.

para telemedicina, inicialmente se concentraram em renovação de receitas, avaliação de necessidade de exames e acompanhamento de condições crônicas. $\mathrm{Na}$ teleconsulta, evolui-se o plano para o Teleagendamento, a data e o responsável pelo caso. O paciente é informado, conforme seu plano, de que receberá uma ligação em determinado dia ou semana. Havendo aumento exponencial de casos de sintomáticos respiratórios, não se faz necessária a ligação para desmarcar consultas presenciais.

O momento atual, com a redução na circulação de pessoas pelas medidas de supressão adotadas no município desde 16/03 (fechamento das instituições de ensino) até 20/03 com as medidas de fechamento de estabelecimentos comerciais, o serviço se organiza para retomar os atributos da longitudinalidade e coordenação, que até o momento (06/04), são ofertados pelo profissional responsável pelos teleatendimentos, independente do vínculo. A segunda semana de abril será de retomada, a partir da lista de pacientes, de usuários prioritários que receberão as ligações telefônicas de seu médico de referência (gestantes, pacientes anticoagulados, diabéticos com glicada acima de 10).

No Gráfico 1 estão descritas a evolução dos casos ao longo das 4 semanas do Plano de Contingência, em relação ao total de atendimentos, as consultas médicas presenciais não $S R$, as consultas presenciais dos $S R$ e as consultas por telefone.

Gráfico 1. Número absoluto, por tipo de consulta, dos usuários atendidos na UBS Santa Cecília, semanas 16 a 20/03; 23 a 27/03 e 30/03 a 03/04/2020. Porto Alegre.RS.

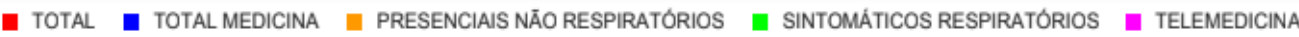

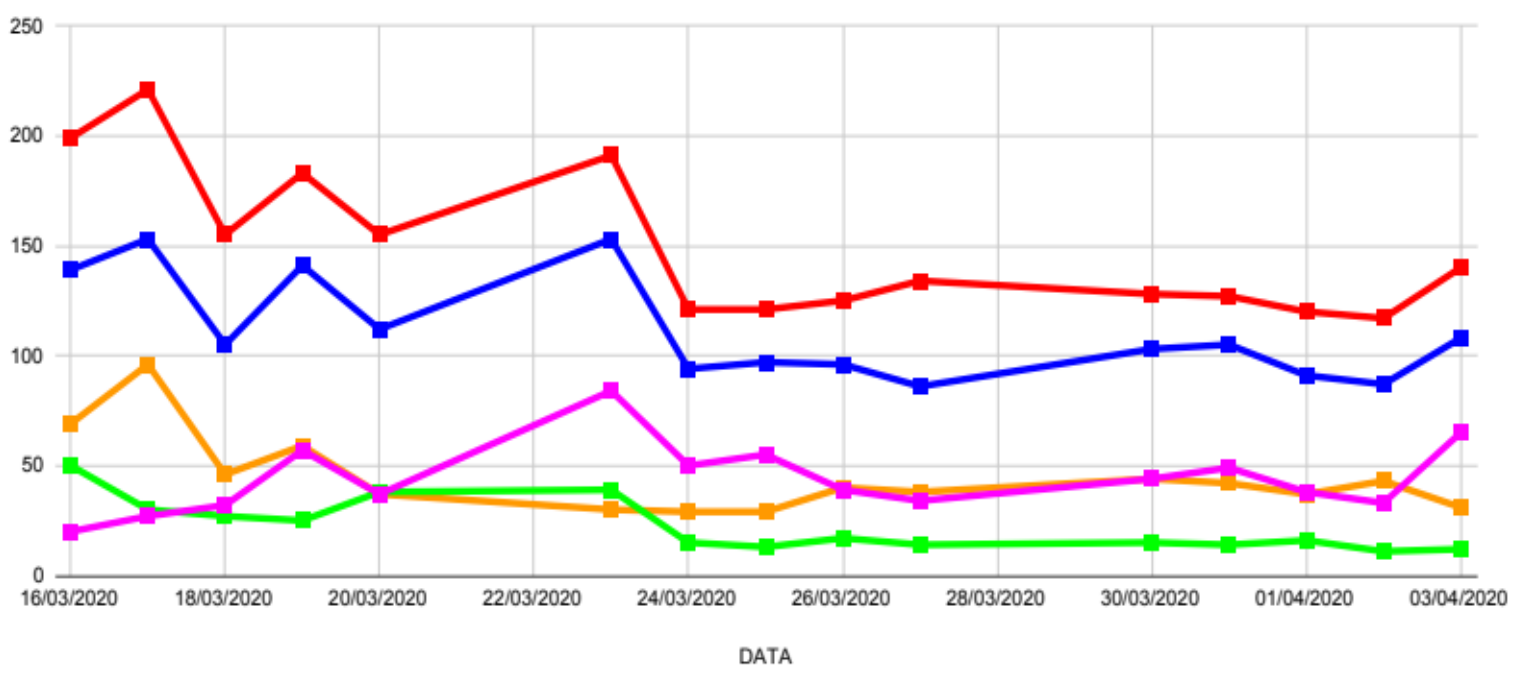

Fonte: Elaboração própria a partir do AGHUse, sistema de informações, HCPA, 2020.

\section{IV) NÃO DEIXAR NINGUÉM}

\section{PARA TRAZ}

Em meio à organização para o enfrentamento da pandemia, a UBS é referência para a imunização de todo o calendário vacinal, com adiamento para após 15 de abril, mas sendo vacinadas as crianças que vêm à unidade, bem como da campanha do H1N1. O plano para a vacina nos idosos foi fazer drive thru, em um dia por três semanas consecutivas, além da vacinação na UBS dos idosos que não tinham veículo. Todos os acamados receberam a vacina no domicílio, e nas próximas duas semanas, os idosos maiores 
Mendonça, C. S.; Rosset, I.; Gonçalves, M. R.; Bastos, C. G. M.; Medeiros, A. F.; Dias, A. V.; Nascimento, F. R.; Pereira, F. V. P.; Pelegrini, G.; Pagnini J.; Leite, L. O.; Arruda, N. M.; Freitas, P.; Firmino, R. B.; Reolom, R.; Tolio, R. P.; Mantese, G. H. A.

de 80 anos também serão vacinados no domicílio.

Além das questões clínicas assistenciais que exigem execução de atividades usualmente realizadas em outros pontos da rede, que estão fechados, o serviço social, juntamente com os ACS estão mobilizados para identificar e apoiar os potenciais beneficiários do programa de renda emergencial para trabalhadores não protegidos por vínculos formais de trabalho, além daqueles já acompanhados pelo Bolsa Família.

Além da necessidade de reforçar os recursos para os cuidados intensivos, torna-se impossível proteger a saúde da população brasileira e reduzir sua morbi-mortalidade na pandemia sem a implantação de processos eficientes e seguros na APS. O Brasil pode mostrar uma resposta diferente de outros países, e demonstrar o significado de ter um sistema de saúde universal com APS forte.

\section{REFERÊNCIAS BIBLIOGRÁFICAS}

BRASIL $^{a}$. Ministério da Saúde. Secretaria de Atenção Primária à Saúde. Protocolo de Manejo Clínico do Coronavírus (Covid-19) na Atenção Primária à Saúde. Versão 6. Brasilia, março de 2020.

BRASIL $^{\mathrm{b}}$. Ministério da Educação. Secretaria de Educação Superior.Comissão Nacional de Residência Médica. Circular no 01/2020. CNRM/CGRS/DDES/SESU/MEC. Assunto: Recomendações quanto ao desenvolvimento das atividades dos Programas de Residência Médica em relação aos planos de enfretamento ao COVID-19.

Kidd, Michael. Australia's primary care COVID-19 response. AJGP.First published online 2 April 2020. doi: 10.31128/AJGP-COVID-02. Acesso em 02/04/2020:

https://www1.racgp.org.au/ajgp/coronavirus/au stralias-primary-care-covid19-response

Liu, Joanne. I've fought epidemics around the world. Now it's Canada that must prepare for the worst. Special to the globe and mail published 6 hours ago updated march 29, 2020.

Lorenzo, Sergio Minué. Contra el coronavirus, más Atención Primaria que nunca. amf.semfyc.com. Acessado em 03/04/2020 em: https://amf-

semfyc.com/web/article_ver.pho?id=2626.

Secretaria Municipal de Saúde. Porto Alegre. Coordenadoria Geral de Vigilância em Saúde. Fluxo de Atendimento aos Casos Suspeitos de novo Coronavírus (Atenção Primária À Saúde).Acessado em 02/04/2020. http://www2.portoalegre.rs.gov.br/cgvs/ 